\title{
A setup for active fault diagnosis
}

\section{Niemann, Hans Henrik}

\section{Published in:}

I E E E Transactions on Automatic Control

Link to article, DOI:

10.1109/TAC.2006.878724

Publication date:

2006

\section{Document Version}

Publisher's PDF, also known as Version of record

Link back to DTU Orbit

\section{Citation (APA):}

Niemann, H. H. (2006). A setup for active fault diagnosis. I E E E Transactions on Automatic Control, 51(9), 1572-1578. https://doi.org/10.1109/TAC.2006.878724

\section{General rights}

Copyright and moral rights for the publications made accessible in the public portal are retained by the authors and/or other copyright owners and it is a condition of accessing publications that users recognise and abide by the legal requirements associated with these rights.

- Users may download and print one copy of any publication from the public portal for the purpose of private study or research.

- You may not further distribute the material or use it for any profit-making activity or commercial gain

- You may freely distribute the URL identifying the publication in the public portal

If you believe that this document breaches copyright please contact us providing details, and we will remove access to the work immediately and investigate your claim 


\section{A Setup for Active Fault Diagnosis}

\author{
Henrik Niemann
}

\begin{abstract}
A setup for active fault diagnosis (AFD) of parametric faults in dynamic systems is formulated in this note. It is shown that it is possible to use the same setup for both open loop systems, closed-loop systems based on a nominal feedback controller as well as for closed-loop systems based on a reconfigured feedback controller. This will make the proposed AFD approach very useful in connection with fault tolerant control (FTC). The setup will make it possible to let the fault diagnosis part of the fault tolerant controller remain unchanged after a change in the feedback controller. The setup for AFD is based on the Youla-Jabr-Bongiorno-Kucera (YJBK) parameterization of all stabilizing feedback controllers and the dual YJBK parameterization. It is shown that the AFD is based directly on the dual YJBK transfer function matrix. This matrix will be named the fault signature matrix when it is used in connection with AFD.
\end{abstract}

Index Terms-Active fault diagnosis (AFD), controller reconfiguration, dual Youla-Jabr-Bongiorno-Kucera (YJBK) parameterization, feedback control systems.

\section{INTRODUCTION}

The interest in using active fault diagnosis in dynamic systems has been increasing doing recent years; see, e.g., [2]-[5], [9], [10], [13], [14].

In the active fault diagnosis approach, parametric faults/system variations are detected by using an auxiliary input vector. The detection/ isolation is derived by using an associated residual vector. One of the advantages by using active fault diagnosis (AFD) is that it can give a much faster detection compared with a passive fault detection and isolation (FDI) approach. In the passive FDI approach, the detection is based on external random inputs or reference inputs on the system. As a result of this, the faults are not detected until they are excited by disturbance or reference inputs. In the active approach, the auxiliary inputs can be designed to excite specified possible faults with a minimal effect on the complete system. The AFD approach has mainly been applied on open-loop systems [2], [3], [5], [13], and [14], but in [9] and [10] the AFD approach has been applied in connection with closed loop systems. It has been shown in [9], [10] that using AFD on a closed loop system is very closely related to the YJBK and the dual YJBK parameterization. It is shown that the fault diagnosis is derived directly on the dual YJBK transfer function. It is also called the fault signature matrix when it is used in connection with AFD.

The focus in [2]-[5] is on the design of optimal auxiliary input signals and evaluation of the measurement signals with respect to a fast model selection/fault detection. The methods can be applied on both open and closed loop systems.

The main focus in this note is to extend the AFD setup for closed loop systems to both open loop systems as well as closed loop systems where the feedback controller is another than the nominal feedback controller. This is important, especially in connection with fault tolerant control (FTC), where the fault diagnosis part of the FTC should still be running after the first fault(s) has been detected/isolated and the controller has been reconfigured. Using the AFD approach, the FDI block will depend on the applied feedback controller. This

Manuscript received May 13, 2005; revised November 11, 2005 and November 28, 2005. Recommended by Associate Editor L. Magni.

The author is with Ørsted•DTU, Automation, Technical University of Denmark, DK-2800 Kgs. Lyngby, Denmark (e-mail: hhn@oersted.dtu.dk).

Digital Object Identifier 10.1109/TAC.2006.878724 means that the FDI block also needs to be changed when the feedback controller is modified.

The main result of this note is that it is possible to apply the same setup for AFD on both open-loop systems as well as on closed loop systems. Based on this setup, it is shown that it is possible to lay down conditions for fault isolation using the AFD approach independent of the applied feedback controller. The limitation here is the maximal number of faults that may occur simultaneuosly, bounded by the number of control signals or measurement signals minus one.

\section{SYSTEM SETUP}

The general systems applied in connection with fault diagnosis and fault tolerant control will now be described. Consider the following generalized nominal system including parametric faults:

$$
\Sigma_{P}:\left\{\begin{array}{l}
z=G_{z w} w+G_{z d} d+G_{z u} u \\
e=G_{e w} w+G_{e d} d+G_{e u} u \\
y=G_{y w} w+G_{y d} d+G_{y u} u
\end{array}\right.
$$

where $d \in \mathcal{R}^{r}$ is a disturbance signal vector, $u \in \mathcal{R}^{m}$ the control input signal vector, $e \in \mathcal{R}^{q}$ is the external output signal vector to be controlled, $y \in \mathcal{R}^{p}$ is the measurement vector, $w \in \mathcal{R}^{k}$ and $z \in \mathcal{R}^{k}$ are external input and output vectors. It is not assumed that it should be a continuous-time system or a discrete-time system. Both types of systems can be handled by using the system setup given by (1).

The connection between the external output and input is given by

$$
w=\theta z
$$

where $\theta$ is a diagonal matrix given by

$$
\theta=\operatorname{diag}\left(\theta_{1}, \ldots, \theta_{i}, \ldots, \theta_{k}\right)
$$

representing the parametric faults in the system. We will use the notation $\theta_{i} \neq 0$ as a short form for $\theta=\operatorname{diag}\left(0, \ldots, 0, \theta_{i}, 0, \ldots, 0\right)$.

Further, let us denote the set of all possible parametric faults by $\mathbf{k}=\{1, \ldots, k\}$. Based on the available information as what parametric faults could occur simultaneously at any time and what cannot, one divides the set of all possible faults into a number of fault sets. Let $\mathbf{k}$ be partitioned into $\ell$ mutually exclusive and exhaustive sets, $\Omega_{i}$, $i=1,2, \ldots, \ell$, including $k_{i}, i=1,2, \ldots, \ell$ faults each fault set. That is, let $\Omega_{i} \cap \Omega_{j}=\varnothing$ for $i \neq j$, and $\Omega_{1} \cup \Omega_{2} \cup \ldots \Omega_{\ell}=\mathbf{k}$; see, e.g., [15]. It is assumed that only faults from a specific fault set $\Omega_{i}$ can occur simultaneoulsy. Let the parametric fault matrix $\theta$ be arranged in such a way that the first $k_{1}$ faults belong to the first fault set $\Omega_{1}$, the next $k_{2}$ faults belong to the second fault set $\Omega_{2}$, etc.

Closing the loop from $w$ to $z$ in $\Sigma_{P}$ by using $\theta$, can be realized by an upper linear fractional transformation (LFT) in $\theta$ given by

$$
\Sigma_{P, \theta}=\mathcal{F}_{u}\left(\Sigma_{P}, \theta\right)
$$

where $\Sigma_{P, \theta}$ is given by

$$
\Sigma_{P, \theta}:\left\{\begin{array}{l}
e=G_{e d}(\theta) d+G_{e u}(\theta) u \\
y=G_{y d}(\theta) d+G_{y u}(\theta) u
\end{array}\right.
$$

Further, let the system be controlled by a stabilizing feedback controller given by

$$
\Sigma_{K}:\{u=K y .
$$




\section{THE YJBK PARAMETERIZATION}

Let a coprime factorization of the system $G_{y u}$ from (1) and a stabilizing controller $K$ from (3) be given by

$$
G_{y u}=N M^{-1}=\tilde{M}^{-1} \tilde{N} \quad K=U V^{-1}=\tilde{V}^{-1} \tilde{U}
$$

where $N, M, \tilde{N}, \tilde{M}, U, V, \tilde{U}$, and $\tilde{V} \in \mathcal{R} \mathcal{H}_{\infty}$ must satisfy the double Bezout equation given by (see [17])

$$
\begin{aligned}
\left(\begin{array}{ll}
I & 0 \\
0 & I
\end{array}\right) & =\left(\begin{array}{cc}
\tilde{V} & -\tilde{U} \\
-\tilde{N} & \tilde{M}
\end{array}\right)\left(\begin{array}{cc}
M & U \\
N & V
\end{array}\right) \\
& =\left(\begin{array}{cc}
M & U \\
N & V
\end{array}\right)\left(\begin{array}{cc}
\tilde{V} & -\tilde{U} \\
-\tilde{N} & \tilde{M}
\end{array}\right) .
\end{aligned}
$$

Based on the previous coprime factorization of the system $G_{y u}$ and the controller $K$, we can give a parameterization of all controllers that stabilize the system in terms of a stable transfer function $Q$, i.e., all stabilizing controllers are given by [17], [18]

$$
K(Q)=(U+M Q)(V+N Q)^{-1}, \quad Q \in \mathcal{R} \mathcal{H}_{\infty}
$$

or by using a left factored form

$$
K(Q)=(\tilde{V}+Q \tilde{N})^{-1}(\tilde{U}+Q \tilde{M}), \quad Q \in \mathcal{R} \mathcal{H}_{\infty}
$$

Using the Bezout equation, the controller given either by (6) or by (7) can be realized as a lower LFT in the parameter $Q$

$$
K(Q)=\mathcal{F}_{l}\left(J_{K}, Q\right)
$$

where $J_{K}$ is given by

$$
J_{K}=\left(\begin{array}{cc}
U V^{-1} & \tilde{V}^{-1} \\
V^{-1} & -V^{-1} N
\end{array}\right)
$$

In the same way, it is possible to derive a parameterization in terms of a stable transfer function $S$ of all systems that are stabilized by one controller, i.e. the dual YJBK parameterization. The parameterization is given by [17]

$$
G_{y u}(S)=(N+V S)(M+U S)^{-1}, \quad S \in \mathcal{R} \mathcal{H}_{\infty}
$$

or by using a left factored form

$$
G_{y u}(S)=(\tilde{M}+S \tilde{U})^{-1}(\tilde{N}+S \tilde{V}), \quad S \in \mathcal{R} \mathcal{H}_{\infty}
$$

An LFT representation of (10) or (11) is given by

$$
G_{y u}(S)=\mathcal{F}_{l}\left(\left(\begin{array}{cc}
N M^{-1} & \tilde{M}^{-1} \\
M^{-1} & -M^{-1} U
\end{array}\right), S\right)=\mathcal{F}_{l}\left(J_{G}, S\right) .
$$

It has been shown in, e.g., [17], that the dual YJBK transfer function $S$ can be calculated by using the primary YJBK parameterization, $S$ is given by

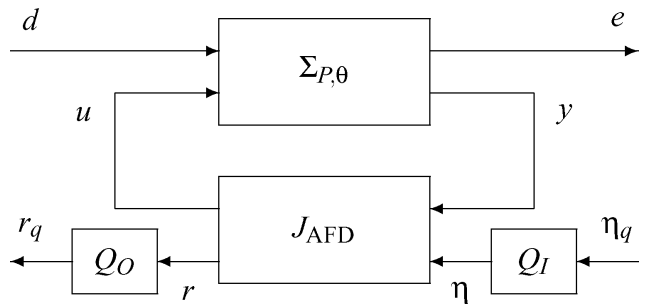

Fig. 1. Setup for AFD in closed loop systems. A pre-filter has been placed at the input vector $\eta$ and a post-filter has been placed at the output vector $r$.

\section{Active Fault Diagnosis for Closed-Loop Systems}

Active fault diagnosis for closed-loop systems has been considered in detail in [9]. An example of using AFD in closed loop systems is given in [10]. Some preliminary results from [9] are given later, together with the introduction of the fault signature matrix.

As a residual vector $r$ in the AFD case, we use the same vector as in the passive FDI approach given by [6]

$$
r_{q}=Q_{O}(\tilde{M} y-\tilde{N} u)=Q_{O} r
$$

where $Q_{O}$ is a stable and proper filter of suitable order. Note that $r$ is also the input vector to the YJBK transfer function.

The residual generator given by (14) is a parameterization of all residual generators in terms of $Q_{O}$, see [6]. The residual generator should be designed such that the residual $r$ or $r_{q}$ vanishes or is close to zero in the fault free case. If the residual is nonvanishing indicate a difference between the real system and the model due to model uncertainty or faults. Statistical methods such as CUSUM or GLR test are used for the evaluation of the residual vector in the passive approach [1]. The fault isolation is obtained, if possible, by designing the residual generator such that some of the residual signals are sensitive to a specific fault and other signals are insensitive to the fault.

Let the auxiliary input vector for AFD be named $\eta$ or $\eta_{q}$ when a filter is included. It can be shown that all residual generators for active fault diagnosis are given by the system setup shown in Fig. 1, where $J_{\mathrm{AFD}}=$ $J_{K}$ is given by (9). $Q_{I}$ and $Q_{O}$ are pre- and postfilters, respectively, that are free for design.

From (9), we have that $\eta$ is equivalent to the output from the YJBK transfer function $Q$. Further, using (13), we get directly that the transfer function from $\eta$ to $r$ is the dual YJBK transfer function, i.e.,

$$
r=S_{\mathrm{cl}}(\theta) \eta
$$

or

$$
r_{q}=Q_{O} S_{\mathrm{cl}}(\theta) Q_{I} \eta_{q}=S_{Q, \mathrm{cl}}(\theta) \eta_{q}
$$

when the pre- and postfilters are included. From [8], we have that the dual YJBK parameter is given by

$$
S_{\mathrm{cl}}(\theta)=\tilde{M} G_{y w} \theta\left(I-T_{z w, \mathrm{cl}} \theta\right)^{-1} G_{z u} M
$$

where $T_{z w, c l}$ is the closed loop transfer function from $w$ to $z$ given by

$$
S=\mathcal{F}_{u}\left(J_{K}, G_{y u}(S)\right)
$$

$$
T_{z w, c l}=G_{z w}+G_{z u} U \tilde{M} G_{y w} .
$$


Fault detection and isolation can then be derived based on $S_{c l}(\theta)$, considered in Section VII. Here, it is shown that the two filters $Q_{I}$ and $Q_{O}$ is applied in connection with fault isolation.

As it can be seen from (16), $S_{\mathrm{cl}}(\theta)$ is very important in connection with AFD. Equivalent to the definition of fault signature for additive faults [17], $S_{\mathrm{cl}}(\theta)$ will be called the fault signature matrix for parametric faults.

In Fig. 1, the auxiliary input $\eta$ is injected directly in the controller. However, it is also possible to inject the $\eta$ at other available signals/vectors. This mean that $\eta$ can, e.g., also be injected in connection with the control input $u$ or the measurement output $y$, i.e., outside the controller. These cases are also included in the setup shown in Fig. 1. Injecting $\eta$ in connection with the measurement vector $y$ is equivalent by using $Q_{I}=\tilde{U}$ in the setup shown in Fig. 1. Equivalent, an injection at the output of the controller can be obtained by using $Q_{I}=\tilde{V}$ in the general setup.

\section{ACtive Fault Diagnosis FOR OPEn LoOP Systems}

Now, let us consider AFD in connection with open loop dynamic systems. The main difference between AFD for closed open systems and open loop systems is the stability aspect. This aspect cannot be considered in connection with an open loop setup for AFD. The stability of the faulty closed loop system can be validated directly by a validation of the stability of the fault signature matrix $S_{\mathrm{cl}}(\theta)$.

AFD for open-loop systems follows almost the same setup as for the closed-loop systems, apart from that the only available input vector is the control input $u$. The residual vector $r$ given by (14) is used as the output vector again.

The open loop system setup can be derived from the closed-loop setup in Fig. 1 by removing the feedback controller. The input injection point for $\eta$ from the closed-loop system does not exist in the open-loop case. Instead, the control input need to be applied. To get the same structure as in the closed-loop case, now let the control input be given by

$$
u=M \eta
$$

and let the residual vector $r$ be given by (14), i.e.,

$$
r=\tilde{M} y-\tilde{N} u=\tilde{M} y-\tilde{N} M \eta .
$$

This together gives $J_{\mathrm{AFD}}$ take the following form:

$$
J_{\mathrm{AFD}}=\left(\begin{array}{cc}
0 & M \\
\tilde{M} & -\tilde{N} M
\end{array}\right)
$$

$J_{\mathrm{AFD}}$ can also be derived from $J_{K}$ in (9) and the double Bezout equation in (5) with $U=\tilde{U}=0$ when the system is open-loop stable.

Based on the system description in (2), the transfer function between the auxiliary input vector $\eta$ and the residual vector $r$ is given by

$$
r=\left(\tilde{M} G_{y u}(\theta)-\tilde{N}\right) M \eta=\tilde{M} G_{y w} \theta\left(I-G_{z w} \theta\right)^{-1} G_{z u} M \eta
$$

which gives directly the following transfer function $S_{\text {open }}(\theta)$ between the input $\eta$ and the residual vector $r$ :

$$
S_{\text {open }}(\theta)=\tilde{M} G_{y w} \theta\left(I-G_{z w} \theta\right)^{-1} G_{z u} M .
$$

Including the two filters in the setup, the transfer function from $\eta_{q}$ to $r_{q}$ take then the following form:

$$
S_{Q, \text { open }}(\theta)=Q_{O} S_{\text {open }}(\theta) Q_{I} .
$$

The transfer function matrix $S_{\text {open }}(\theta)$ given by (19) is completely equivalent to the fault signature matrix $S_{\mathrm{cl}}(\theta)$ given by (16) for the closed-loop case. As a consequence of this equivalence between the open-loop and the closed-loop case, it is therefore natural to name Sopen given by (19) as the open-loop fault signature matrix.

\section{Active Fault Diagnosis for ReConfigured Closed LOOP SYSTEMS}

In connection with FTC, the feedback controller is normally reconfigured after a parametric fault has been detected and/or isolated in the system [11]. This reconfiguration can be done by using the YJBK transfer function $Q$. The open loop transfer function from $\eta$ to $r$ in Fig. 1 is closed by a $Q$. A detailed description of controller reconfiguration by using the YJBK parameterization is given in [11].

Including a feedback controller $Q$ from $r$ to $\eta, J_{\mathrm{AFD}}$ takes then the following form:

$$
J_{\mathrm{AFD}}=\left(\begin{array}{cc}
K(Q) & (\tilde{V}+Q \tilde{N})^{-1} \\
(V+N Q)^{-1} & -(V+N Q)^{-1} N
\end{array}\right) .
$$

Based on the $J_{\mathrm{AFD}}$ given in (21), it is possible to calculate the transfer function from $\eta$ to $r$ given by $S_{\text {rec }}(\theta)$. The transfer function is given by

$$
S_{\mathrm{rec}}(\theta)=\tilde{M} G_{y w} \theta\left(I-T_{z w, \mathrm{cl}}(Q) \theta\right)^{-1} G_{z u} M
$$

where

$$
T_{z w, \mathrm{cl}}(Q)=T_{z w, \mathrm{cl}}+G_{z u} M Q \tilde{M} G_{y w} .
$$

Again, including the pre- and postfilter at the input $\eta$ and output $r$ gives the following transfer function:

$$
S_{Q, \mathrm{rec}}(\theta)=Q_{O} S_{\mathrm{rec}}(\theta) Q_{I}
$$

In line with the nominal feedback case and the open-loop case, $S_{\mathrm{rec}}(\theta)$ is then the fault signature matrix for the reconfiguraded feedback system.

Further, note that the fault signature matrix given by (22) can also be written as

$$
S_{\mathrm{rec}}(\theta)=S_{\mathrm{cl}}(\theta)\left(I-Q S_{\mathrm{cl}}(\theta)\right)^{-1}
$$

where $S_{\mathrm{cl}}(\theta)$ is the fault signature matrix for the nominal controller case given by (16). (24) can be derived directly by using the fact that the transfer function from $\eta$ to $r$ given by $S_{\mathrm{cl}}(\theta)$ is closed by using $Q$.

The above results are also valid for more direct controller changes, i.e., where a nominal feedback controller $K$ is directly changed by a new controller $K_{1}$ without using the YJBK architecture. Using the results from [12], it is shown how to calculate the equivalent YJBK transfer function for such a controller switching. This means that there will always be a related YJBK transfer function that can describe a controller change.

\section{AFD BASED ON THE FAUlt SignATURE MATRIX}

As it can be seen from this, the fault signature matrix $S(\theta)$ has the same structure for all three cases. The general fault signature matrix $S$ can be given by

$$
S(\theta)=\tilde{M} G_{y w} \theta(I-T \theta)^{-1} G_{z u} M
$$


where $T$ is an open-loop or a closed-loop transfer function from $w$ to $z$ depending on the case. The general fault signature matrix given by (25) then takes the following form depending on $T$ :

$$
\begin{array}{ll}
S=S_{\text {open }} & \text { for } T=G_{z w} \\
S=S_{\mathrm{cl}} & \text { for } T=T_{z w, \mathrm{cl}} \\
S=S_{\mathrm{rec}} & \text { for } T=T_{z w, \mathrm{cl}}+G_{z u} M Q \tilde{M} G_{y w} .
\end{array}
$$

Note that this requires that the applied $M$ and $\tilde{M}$ in the open-loop case satisfy the double Bezout equation in (5) for the nominal feedback controller.

Fault detection based on $S(\theta)$ given by (25) is given directly by

- Fault detection

$$
\begin{array}{ll}
S(\theta)=0, & \text { for } \theta=0 \\
S(\theta) \neq 0, & \text { for } \theta \neq 0 .
\end{array}
$$

By a simple test, it is possible to detect parametric faults. This can be done by using a periodic auxiliary input $\eta$ on the system and, e.g., a dedicated CUSUM test on the residual vector $r$. For more details, see Section VIII and [10].

The fault isolation case is more complicated. The main reason is that in general all elements in $S(\theta)$ will depend on all parametric faults. This means that it will be impossible to isolate the single parametric faults by evaluating the single elements in $S(\theta)$, i.e., direct fault isolation. Further, it will also depend on which parametric faults that can occur simultaneously and which cannot.

Let us consider the fault signature matrix given by (25), where the pre- and postfilters $Q_{I}$ and $Q_{O}$, have been included. The design of $Q_{I}$ and $Q_{O}$ must be done in such a way that an isolation (separation) of the parametric faults can be done directly by considering $S_{Q}(\theta)$, when it is possible. Using the fact that both $\tilde{M} G_{y w}$ and $G_{z u} M$ are two stable transfer functions, it is possible to design $Q_{I}$ and $Q_{O}$ in such a way that

$$
G_{z u} M Q_{I}=\left(\begin{array}{l}
\Xi_{I} \\
H_{I}
\end{array}\right) \quad Q_{O} \tilde{M} G_{y w}=\left(\begin{array}{ll}
\Xi_{O} & H_{O}
\end{array}\right)
$$

where $\Xi_{I}$ and $\Xi_{O}$ are two stable diagonal matrices of dimension $m \times m$ and $p \times p$, respectively, and $H_{I}, H_{O}$ are two stable transfer matrices of suitable dimension. Note that if $G_{z u} M$ is right invertible, i.e., $k \leq$ $m$, we can obtain a diagonalization of $G_{z u} M$ by the design of $Q_{I}$. Equivalent, if $\tilde{M} G_{y w}$ is left invertible, i.e. $k \leq p$, a diagonalization of $\tilde{M} G_{y w}$ can be obtained by the design of $Q_{O}$.

Using the $Q_{I}$ and $Q_{O}$ satisfying (26) in (25) gives directly

$$
S_{Q}(\theta)=\left(\begin{array}{ll}
\Xi_{O} & H_{O}
\end{array}\right) \theta(I-T \theta)^{-1}\left(\begin{array}{l}
\Xi_{I} \\
H_{I}
\end{array}\right) .
$$

As it can be seen from (27), it will not in the general case be possible to obtain a complete diagonalization of the input and/or output transfer functions. If it is not possible to diagonalize either the input or the output transfer functions by the design of $Q_{I}$ or $Q_{O}$, it will not be possible to obtain a complete fault separation in $S_{Q}(\theta)$.

If $p \geq 2$ or $m \geq 2$, fault isolation for some type of fault sets is still possible. Assume that $p \geq m$, i.e., the number of measurement signals is larger than or equal to the number of control signals. It is then possible to get fault isolation if the number of faults $k_{i}$ in the fault sets $\Omega_{i}, i=1, \ldots l$ is maximal $p-1$.

Using a $Q_{O, 1}$ satisfying (26) with respect to the first fault set $\Omega_{1}$ gives the following fault signature matrix:

$$
S_{Q, \Omega_{1}}(\theta)=\left(\Xi_{O} \quad H_{O}\right) \theta(I-T \theta)^{-1} G_{z u} M Q_{I} .
$$

Let $S_{Q, \Omega_{1}}(\theta)$ be partitioned into

$$
S_{Q, \Omega_{1}}(\theta)=\left(\begin{array}{c}
S_{Q, \Omega_{1}, 1}(\theta) \\
S_{Q, \Omega_{1}, 2}(\theta)
\end{array}\right)=\left(\begin{array}{c}
S_{Q, \Omega_{1}, 1,1}(\theta) \\
\vdots \\
S_{Q, \Omega_{1}, 1, k_{1}}(\theta) \\
S_{Q, \Omega_{1}, 2}(\theta)
\end{array}\right)
$$

where $S_{Q, \Omega_{1}, 1}(\theta)$ is the first $k_{1}$ rows in $S_{Q, \Omega_{1}}(\theta)$ and $S_{Q, \Omega_{1}, 2}(\theta)$ is the last $p-k_{1}$ rows in $S_{Q, \Omega_{1}}(\theta)$.

Based on the partition of $S_{Q}$ in (29), it is possible to isolate the fault set wherein faults has occurred. Assume that faults has occurred in the system, we have then that the faults belong to the fault set $\Omega_{1}$ if and only if

$$
S_{Q, \Omega_{1}, 2}(\theta)=0, \quad \theta \neq 0, \quad \theta \in \Omega_{1}
$$

Further, the faults belong to another fault set than $\Omega_{1}$ if and only if

$$
S_{Q, \Omega_{1}, 2}(\theta) \neq 0, \quad \theta \neq 0, \quad \theta \notin \Omega_{1}
$$

In the same way, a $Q_{O, 2}$ is designed with respect to the second fault, etc.

All together, we have a three step procedure: First, a fault detection. Second, a fault set isolation and finally a fault isolation in the specific fault set. The three steps are as follows.

- Fault detection

$$
\begin{array}{ll}
S_{Q}(\theta)=0 & \text { for } \theta=0 \\
S_{Q}(\theta) \neq 0 & \text { for } \theta \neq 0 .
\end{array}
$$

- Fault isolation of the fault set $\Omega_{i}$

$$
\begin{array}{ll}
S_{Q, \Omega_{i}, 2}(\theta)=0 & \theta \neq 0, \theta \in \Omega_{i} \\
S_{Q, \Omega_{i}, 2}(\theta) \neq 0 & \theta \neq 0, \theta \notin \Omega_{i} .
\end{array}
$$

- Fault isolation of the faults in the fault set $\Omega_{i}$.

The $j^{\prime}$ th fault in the fault set $\Omega_{i}$ occurs in the system if and only if:

$$
S_{Q, \Omega_{i}, 1, j}(\theta) \neq 0 \quad \theta_{j} \neq 0, \quad j=1, \ldots, k_{i} .
$$

The $j^{\prime}$ th fault in the fault set $\Omega_{i}$ does not occur in the system if and only if

$$
S_{Q, \Omega_{i}, 1, j}(\theta)=0 \quad \theta_{j}=0, \quad j=1, \ldots, k_{i}
$$

where $S_{Q, \Omega_{i}, 1, j}(\theta)$ is the $j$ 'th row of $S_{Q, \Omega_{i}, 1}(\theta)$ given in (29).

From the definition of the fault sets, the fault related to the last row(s) in $S_{Q, \Omega_{i}}(\theta)$ will not belong to the specific fault set. Further, note that faults which do not belong to the specified fault set will always result in a nonzero row(s) of $S_{Q, \Omega_{i}, 2}(\theta)$ and maybe also in nonzero rows of $S_{Q, \Omega_{i}, 1}(\theta)$. The reason is that if the faults in $\theta$ are isolable, then faults that does not belong to the specific fault set will affect the nondiagonal matrix $H_{0}$. As a result of this, a single fault that does not belong to the specific fault set can, therefore, affect all rows in $S_{Q, \Omega_{i}, 1}(\theta)$.

It is important to point out that the above AFD allow to detect and isolate an unlimited number of faults, as long as the faults can be partitioned into fault sets that can be isolated.

It should be pointed out that the principle behind the above AFD scheme can also be applied in connection with FDI for additive faults. In [16], a fault signal estimation scheme for additive faults based on this concept has been applied. 


\section{DISCUSSION OF DESIGN OF AUXILIARY INPUT}

The selection of the auxiliary input $\eta$ (or $\eta_{q}$ ) and the evaluation of the residual vector $r$ (or $r_{q}$ ) has not been considered in this note. Some comments are given in this section with respect to these aspects.

The design of optimal auxiliary input vectors has been considered in details in [2]-[5]. These methods can be applied directly in connection with the results presented in this note. Also the evaluation of the residual vectors can be done by using the methods from [2]-[5] or by using a CUSUM or GLR test, [1].

Another way to select the auxiliary input vector has been done in [10]. Here, a sinusoid signal has been applied as the auxiliary input signal. The advantage for using such a simple signal is that the detection is quite simple. Here, we only need to evaluate the residual vector at a signal frequency, the frequency of the sinusoid. This is a simple way to detect if a fault signature matrix is zero or non-zero. Combining this with the separation results given in this note, it is possible both to detect and isolate parametric faults in a simple way.

A disadvantage by using a simple signal as a sinusoid signal as the auxiliary input signal is that the detection time might be quite large. The detection time will depend on both the selected frequency for the sinusoid signal as well as on the amplitude. The amplitude and frequency must be selected with respect to the effect from the auxiliary input is maximized on the residual signal and minimized on the external output vector $e$. The application of simple auxiliary input vectors is also considered in the example given in Section IX.

\section{EXAMPLE}

The example is a two mass spring system with two measurement signals given by $G_{y u}=(A, B, C),[18]$

$$
\begin{aligned}
(A \mid B) & =\left(\begin{array}{cccc|c}
0 & 0 & 1 & 0 & 0 \\
0 & 0 & 0 & 1 & 0 \\
-1 & 1 & -0.2 & 0.2 & 1 \\
0.5 & -2.5 & 0.1 & -0.15 & 0
\end{array}\right) \\
C & =\left(\begin{array}{llll}
1 & 0 & 0 & 0 \\
0 & 1 & 0 & 0
\end{array}\right) .
\end{aligned}
$$

An observer based feedback controller is applied to the system with the following state feedback gain $F$ and observer gain $L$ :

$$
\begin{aligned}
& F=\left(\begin{array}{llll}
-0.6886 & -1.1124 & -0.6288 & 0.0837
\end{array}\right) \\
& L=\left(\begin{array}{cc}
-8.3733 & 16.2211 \\
-0.3220 & -5.2795 \\
2.1388 & -70.0381 \\
53.0028 & -195.6965
\end{array}\right) \text {. }
\end{aligned}
$$

Two sensor faults can occur in the system. The two sensor faults can occur simultaneously. The system is then given by

$$
G_{y u}(\theta)=(I+\theta) G_{y u}
$$

where $\theta=\operatorname{diag}\left(\theta_{1}, \theta_{2}\right)$ with $\theta_{1} \in[-1,0]$ and $\theta_{2} \in[-1,0] . \theta_{1}=-1$ and $\theta_{2}=-1$ indicate a complete loss of sensors 1 and 2, respectively.

Now let us consider both fault detection and fault isolation in the open loop system as well as in the closed-loop system. From the definition of the fault signature matrix, it is clear that $S(\theta)$ is nonzero for all values of $\theta \neq 0$. This is the case for both the open-loop as well as for the closed-loop case.

The fault isolation case is more complex. However, when the number of faults is equal to the number of measurement signals, a complete separation of the fault signature matrix is possible.

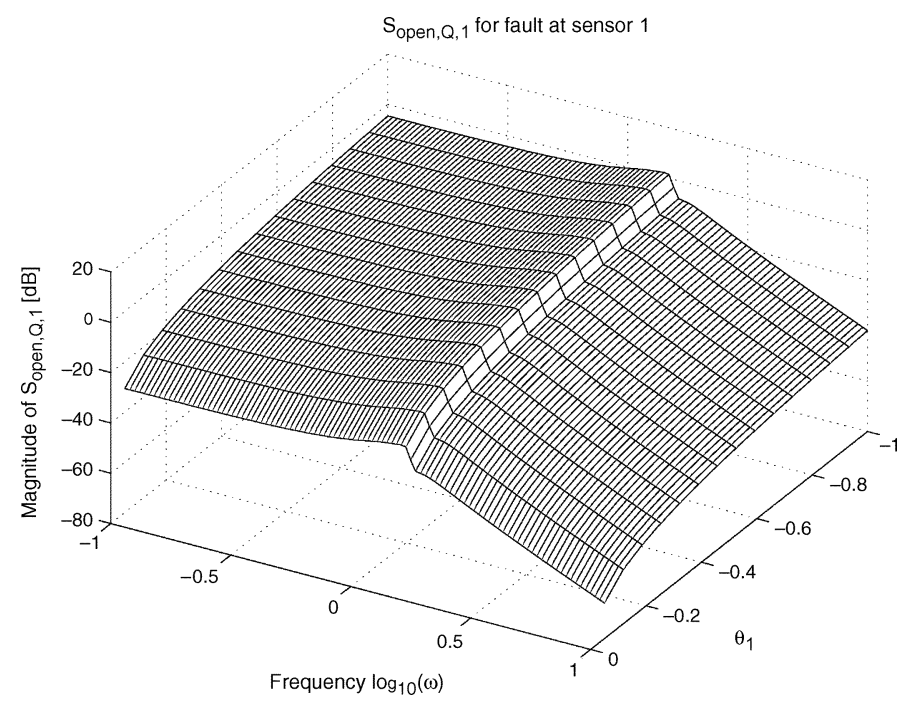

Fig. 2. Bode plot of $S_{o p e n, Q, 1}(\theta)$ as a function of $\theta_{1}$, i.e., fault at the first sensor.

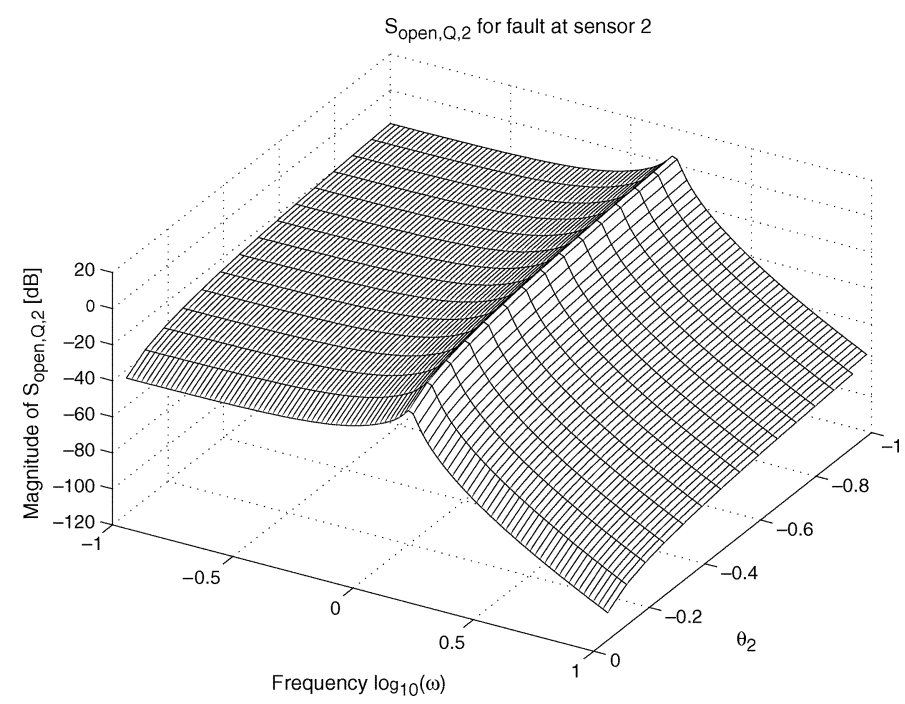

Fig. 3. Bode plot of $S_{o p e n, Q, 2}(\theta)$ as a function of $\theta_{2}$, i.e., fault at the second sensor.

From the system setup, we have that $\tilde{M} G_{y w}=\tilde{M}$ because $G_{y w}=$ $I$. A simple choice for $Q_{O}$, such that (26) is satisfied, is to use $Q_{O}=$ $\tilde{M}^{-1}$ because $\tilde{M}^{-1}$ is stable. By using this output weight function, the fault signature matrix is then given by

$$
S_{Q}(\theta)=\left(\begin{array}{l}
S_{Q, 1}\left(\theta_{1}\right) \\
S_{Q, 2}\left(\theta_{2}\right)
\end{array}\right)
$$

i.e., a complete separation between the two parametric faults. The Bode plots of the fault signature matrix $S_{Q}(\theta)$ is shown for both the openloop case as well as for the closed-loop case. The open-loop case is shown in Figs. 2 and 3, and the closed-loop case is shown in Figs. 4 and 5, respectively. The open-loop case is derived by using the same coprime factorization as for the closed-loop system.

From these figures, it is clear that the feedback controller has an effect on the fault signature matrix. However, the applied feedback controller does not change the structure of the $S_{Q}(\theta)$ so much in this example. The fault signature matrix has an maximum for both the open loop as well as for the closed loop system around $\omega=1.0 \mathrm{rad} / \mathrm{s}$. 


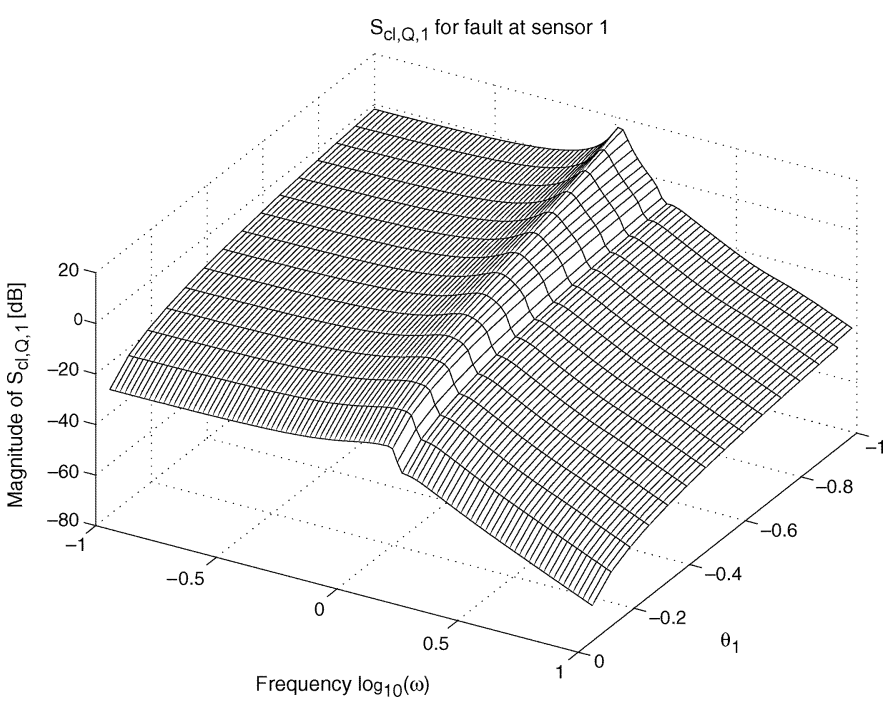

Fig. 4. Bode plot of $S_{c l, Q, 1}(\theta)$ as a function of $\theta_{1}$, i.e., fault at the first sensor.

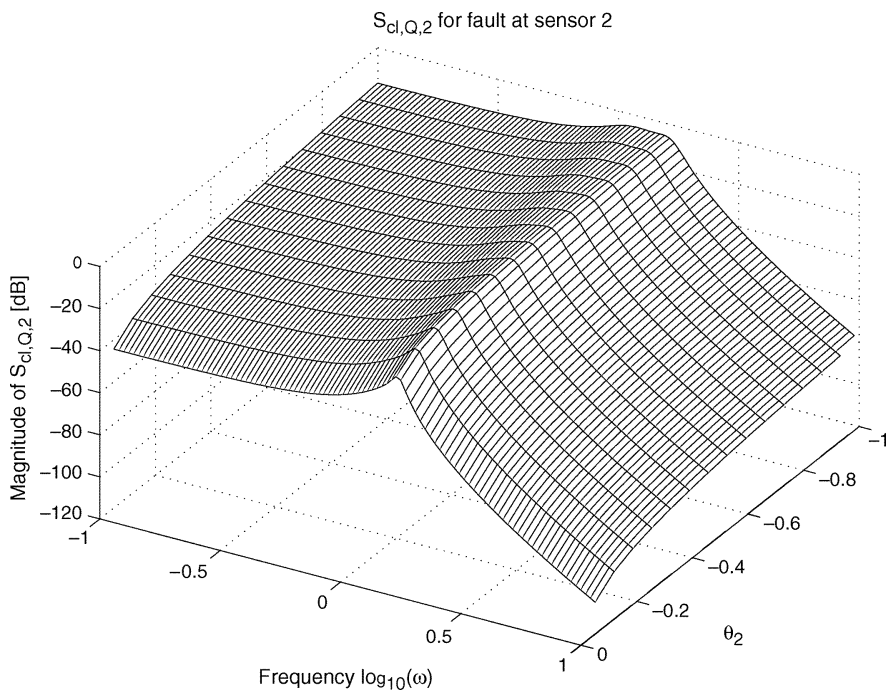

Fig. 5. Bode plot of $S_{c l, Q, 2}(\theta)$ as a function of $\theta_{2}$, i.e., fault at the second sensor.

The system is now simulated. Random uniform distributed disturbance with maximal gain 0.05 has been been included in the control signal $u$. The auxiliary input vector has been selected as

$$
\eta=0.05 \sin \left(\omega_{0} t\right) \quad \omega_{0}=0.02 \mathrm{rad} / \mathrm{s} .
$$

The amplitude and the frequency are selected with respect to minimize the effect from $\eta$ on the output $e=y$ of the system and maximize the detection/isolation time. From Figs. 2-5, a natural selection of the frequency of the $\sin$ function is $1.0 \mathrm{rad} / \mathrm{s}$, i.e., where the amplitudes of $S_{Q}$ are maximal. A more detailed analysis show that it will give a much faster fault detection/isolation by using a much smaller frequency for $\eta$. Further, the effect on the output from $\eta$ will also be reduced.

A CUSUM or GLR detector has not been included in the simulation. To verify detection and isolation in the simulations, the following illustration signal $\delta$ has been applied:

$$
\delta=\left|\int_{0}^{T} r e^{j \omega_{0} t} d t\right| .
$$

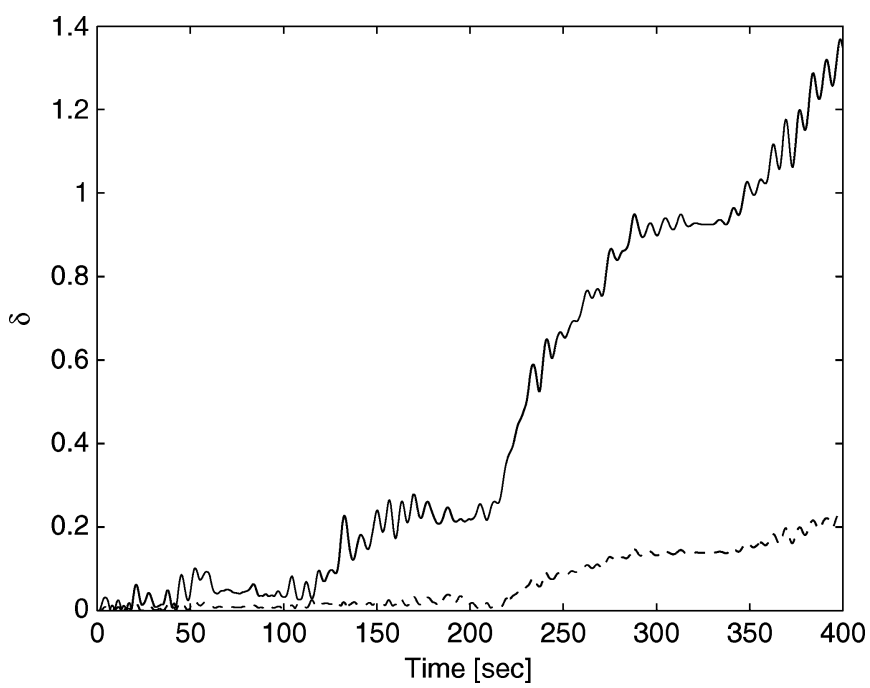

Fig. 6. Illustration signals $\delta_{1}$ (solid line) for fault at sensor no. 1 and $\delta_{2}$ (dashed line) for sensor no. 2, when the system is simulated in closed loop.

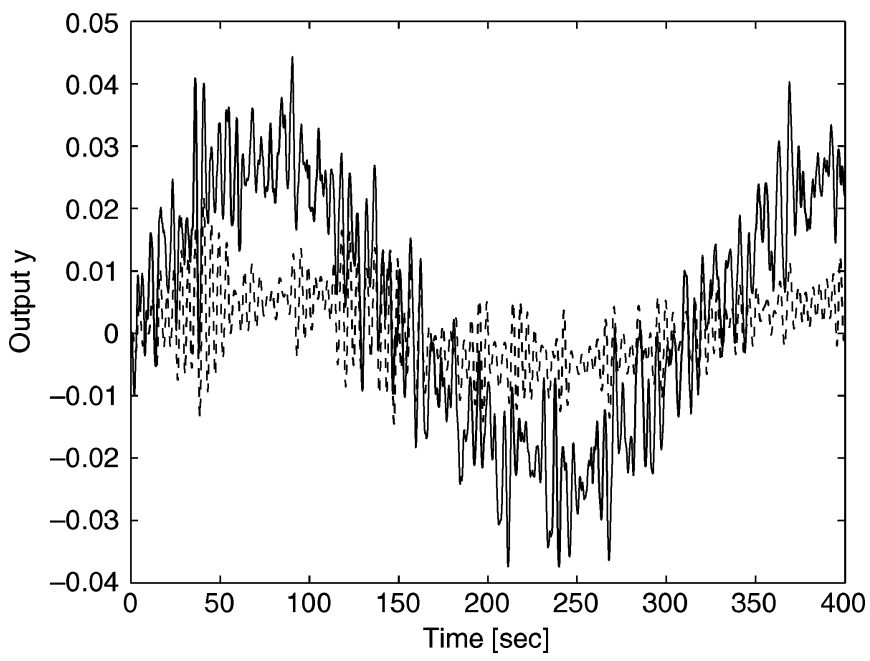

Fig. 7. Output signals $y_{1}$ (solid line) and $y_{2}$ (dashed line) when the system is simulated in closed loop.

The simulations are derived in the following way: The auxiliary input signal is started at $t=0$. A parametric fault given by $\theta_{1}=-0.25$ at sensor no. 1 is introduced at $t=100 \mathrm{sec}$. and a parametric fault given by $\theta_{2}=-0.25$ at sensor no. 2 is introduced at $t=200 \mathrm{sec}$.

The illustration signals $\delta$ are shown in Fig. 6 for the closed-loop system (a simulation of the open loop system gives an equivalent set of curves). It can be seen from the curves that $\delta_{1}$ start increasing just after the fault at sensor no. 1 has occur at $t=100 \mathrm{~s}$. Further, fault at sensor no. 1 does not affect $\delta_{2}$. Equivalently, $\delta_{2}$ stay small until fault at sensor no. 2 occur at $t=200 \mathrm{sec}$. At the same, fault at sensor no. 2 does not affect $\delta_{1}$. In Fig. 7, the effect from the auxiliary signal $\eta$ in the output $y$ is shown for the closed-loop case.

Simulation of both the open loop system and by uing a reconfigurated controller will give the same results.

\section{CONCLUSION}

A setup for active fault diagnosis of parametric faults has been formulated. In connection with the setup, the fault signature matrix has been introduced and the relation with the dual YJBK transfer function has been considered. 
It has been shown that if fault isolation can be derived based on a separation in the fault signature matrix, the fault isolation will not be affected by the inclusion of a feedback controller in the loop, or the feedback controller is reconfigured by using the YJBK parameterization.

In the case where an exact decoupling of the parametric faults via $S(\theta)$ is not possible, the fault isolation must be carried out through the difference in the dynamic characteristic from the parametric faults. In this case, the fault isolation will depend strongly on the applied feedback controller.

\section{REFERENCES}

[1] M. Basseville and I. V. Nikiforov, Detection of Abrupt ChangesTheory and Application. Upper Saddle River, NJ: Prentice-Hall, 1993.

[2] S. L. Campbell, K. G. Horton, and R. Nikoukhah, "Auxiliary signal design for rapid multi-model identification using optimization," Automatica, vol. 38, pp. 1313-1325, 2002.

[3] S. L. Campbell, K. G. Horton, R. Nikoukhah, and F. Delebecque, "Rapid model selection and the separability index," in Proc. Safeprocess 2000, Budapest, Hungary, 2000, pp. 1187-1192.

[4] S. L. Campbell and R. Nikoukhah, Auxiliary Signal Design for Failure Detection. Princeton, NJ: Princeton Univ. Press, 2004.

[5] _ _ "Software for auxiliary signal design," in Proc. Amer. Control Conf., Boston, MA, 2004, pp. 4414-4419.

[6] P. M. Frank and X. Ding, "Frequency domain approach to optimally robust residual generation and evaluation for model-based fault diagnosis," Automatica, vol. 30, pp. 789-804, 1994.

[7] M. Massoumnia, "A geometric approach to the synthesis of failure detection filters," IEEE Trans. Autom. Control, vol. AC-31, no. 9, pp. 839-846, Sep. 1986.

[8] H. H. Niemann, "Dual Youla parameterization," Proc. Inst. Elect. Eng. Control Theory Appl., vol. 150, no. 5, pp. 493-497, 2003.

[9] —_, "Fault tolerant control based on active fault diagnosis," in Proc. Amer. Control Conf., Portland, OR, 2005, pp. 2224-2229.

[10] H. H. Niemann and N. K. Poulsen, "Active fault diagnosis in closedloop systems," in Proc. 16th IFAC World Congr., Prague, Czech Republic, 2005, p. 6p, paper no. 02333.

[11] H. H. Niemann and J. Stoustrup, "An architecture for fault tolerant controllers," Int. J. Control, vol. 78, no. 14, pp. 1091-1110, 2005.

[12] H. H. Niemann, J. Stoustrup, and R. B. Abrahamsen, "Switching between multivariable controllers," Optim. Control Appl. Meth., pp. 51-66, 2004.

[13] R. Nikoukhah, "Innovations generation in the presence of unknown inputs: Application to robust failure detection," Automatica, vol. 30, pp. 1851-1867, 1994.

[14] R. Nikoukhah, S. L. Campbell, and F. Delebecque, "Detection signal design for failure detection: a robust approach," Int. J. Adapt. Control Signal Process., vol. 14, pp. 701-724, 2000.

[15] A. Saberi, A. A. Stoorvogel, P. Sannuti, and H. H. Niemann, "Fundamental problems in fault detection and identification," Int. J. Robust Nonlinear Control, vol. 10, no. 14, pp. 1209-1236, 2000.

[16] A. A. Stoorvogel, H. H. Niemann, A. Saberi, and P. Sannuti, "Optimal fault signal estimation," Int. J. Robust Nonlinear Control, vol. 12, no. 8, pp. 697-727, 2002.

[17] T. T. Tay, I. M. Y. Mareels, and J. B. Moore, High Performance Control. Boston, MA: Birkhäuser, 1997.

[18] K. Zhou, Essentials of Robust Control. Upper Saddle River, NJ: Prentice-Hall, 1998.

\section{Fault Accommodation for Nonlinear Dynamic Systems}

Bin Jiang, Marcel Staroswiecki, and Vincent Cocquempot

\begin{abstract}
This note investigates process fault accommodation in a class of nonlinear continuous-time systems. A new fault estimation module, based on an adaptive estimator, is first proposed. The fault tolerant controller is constructed to compensate for the effect of the faults by stabilizing the closed-loop system. A flexible joint robotic example is given to illustrate the efficiency of the proposed approach.
\end{abstract}

Index Terms-Active fault-tolerant control (FTC), adaptive estimator, fault estimation, nonlinear continuous-time systems.

\section{INTRODUCTION}

In complex systems, dependability is as important as performances. Faults may drastically change the system behavior, ranging from performance degradation to instability. Fault tolerant control (FTC) is needed in order to reach the system objectives, or if this turns to be impossible, to assign new (achievable) objectives to avoid catastrophic behaviors. While passive fault tolerance considers systems faults as a special kind of uncertainties, active fault tolerance is based on fault detection and isolation (FDI).

Active FTC is obtained by fault accommodation (FA), which controls the faulty system, or by system reconfiguration (SR), which controls the healthy (reconfigured) part of the system [27]. Therefore, in FA, the FDI module must detect and isolate the faults, as well as estimate them, in order for the FTC algorithm to determine the appropriate control law [19]. "Appropriate" is meant with respect to given objectives which depend on the application, but which in all cases must preserve stability. During the last decades, different approaches to FA have been reported: pseudo-inverse [10], adaptive control [4], [14], [29], eigenstructure assignment [15], and compensation via additive input design [22]. The survey papers [2] and [23] give the state of the art in the field of FTC, and recent advances are reported in [3].

In most of the existing literature, FDI is addressed separately [5], [11], although some works provide integrated FDI and FTC schemes; for example, see [33] and [34] for FA, and [9] and [21] for integration of FTC and FDI. In this note, a framework for the design of integrated FDI and FTC for a class of nonlinear systems is provided. First, an adaptive estimator is constructed for the simultaneous estimation of the system state and the process fault(s). The fault estimate is then used to construct a fault-tolerant controller, which stabilizes the closed-loop system. Compared to existing works, the contributions of this note are in two aspects: i) A new approach for the estimation of time-varying process faults in a class of nonlinear systems; and ii) A composite fault

Manuscript received June 18, 2003; revised August 8, 2004, December 3, 2005, and March 2, 2006. Recommended by Associate Editor M. Demetriou. This work was supported in part by the National Natural Science Foundation of China under Grant 60574083, by the Key Laboratory of Process Industry Automation, by the State Education Ministry of China, and by the Innovation Scientific Research fund of NUAA.

B. Jiang is with the College of Automation Engineering, Nanjing University of Aeronautics and Astronautics, Nanjing 210016, P.R. China (e-mail: binjiang@nuaa.edu.cn).

M. Staroswiecki is with SATIE-CNRS-UMR8029, Ecole Normale Supérieure de Cachan 61, F-94235 Cachan cedex, France (e-mail: marcel. staroswiecki@univ-lille1.fr).

V. Cocquempot is with LAGIS-CNRS-UMR 8146, Université des Sciences et Technologies de Lille, 59655 Villeneuve d'Ascq Cedex, France (e-mail: vincent. cocquempot@univ-lille1.fr).

Digital Object Identifier 10.1109/TAC.2006.878732 